\title{
LOW COMPLEXITY ITERATIVE MLSE EQUALIZATION OF M-QAM SignALS IN EXTREMELY LONG RAYLEIGH FADING CHANNELS
}

\author{
H.C. Myburgh ${ }^{1}$, Student Member, IEEE, J.C. Olivier ${ }^{2}$
}

\begin{abstract}
This work proposes a neural network based iterative Maximum Likelihood Sequence Estimation (MLSE) equalizer, able to equalize signals in M-arry Quadrature Amplitude Modulation (M-QAM) modulated systems in a mobile fading environment with extremely long channels. Its computational complexity is linear in the data block length and approximately independent of the channel memory length, whereas conventional equalization algorithms have computational complexity linear in the data block length but exponential in the channel memory length. Its performance is compared to the Viterbi MLSE equalizer for short channels and it is shown that the proposed equalizer has the ability to equalize M-QAM signals in systems with hundreds of memory elements, achieving matched filter bound performance with perfect channel state information (CSI) knowledge in uncoded systems. The proposed equalizer is evaluated in a frequency selective Rayleigh fading environment.
\end{abstract}

Index Terms: Equalization, computational complexity, neural network, MLSE.

\section{INTRODUCTION}

Traditional MLSE equalizers used to mitigate the effect of multipath in single-carrier communication systems, based on the Viterbi Algorithm (VA) [1], [2] and the Maximum A Posteriori Probability (MAP) algorithm [3], have computational complexities linear in the data block length and exponential in the channel memory length. When these equalizers are used in systems that have large delay spreads, their computational complexities become extremely high, rendering them unfeasible in practical systems. The VA and MAP algorithms can therefore not be applied in systems with moderate to high bandwidth, due to the exponential relationship between the channel delay spread and the computational complexity.

It is often necessary to revert to suboptimal equalization techniques in order to alleviate the computational strain of these optimal algorithms. Decision feedback equalization (DFE) techniques are often used, where a Minimum Mean Square Error (MMSE) feed-forward filter is used in conjunction with a feedback filter to perform symbol-by-symbol detection. [5] proposes a Delayed Decision Feedback Equalization (DDFE) [4] technique where the first few taps are equalized using a reduced state trellis, while a feedback loop is used to mitigate the inter-symbol interference (ISI) caused by the rest of the taps in the
CIR. DFE techniques are suboptimal, since noise enhancement limits their performance.

Two popular techniques often used to overcome the ISI introduced by long fading channels are Orthogonal Frequency Division Multiplexing (OFDM) [6] and Turbo Equalization [7]. By exploiting the orthogonality properties of the Fourier matrix, OFDM modulation can almost completely eliminate the effect of multipath on the system performance while maintaining trivial per symbol complexity. OFDM, however, is very susceptible to Doppler shift, suffers from a large peak-to-average power ratio (PAPR), and requires large overhead when the channel delay spread is very long compared to the symbol interval. The process of Turbo Equalization uses an iterative equalizer/decoder structure, comprised of a sub-optimal low complexity soft output MMSE equalizer and a soft-in-soft-out MAP decoder. By iterating the system a number of times, the bit-error rate (BER) per- formance can be increased greatly, but at the cost of computational complexity and a substantial overhead due to errorcorrection coding.

In this paper a neural network based iterative MQAM MLSE equalizer is proposed with performance comparable to that of the VA and MAP algorithms, but with computational complexity linear in the data block length $N$ and almost independent of the channel length $L$. Results show that the proposed equalizer can equalize 4-QAM signals is systems with channel lengths of $L=200$ near-optimally. It also is shown that a signal transmitted by a system employing 16QAM modulation, with a channel length of $L=400$, can be equalized by the proposed equalizer with relative ease, while for Viterbi MLSE the trellis would have required some $16^{399}$ states per transmitted symbol in the trellis.

The paper is organized as follows. The iterative MLSE equalizer is presented in Section 2 while the the multilevel neuron is discussed in section 3, followed by a discussion on optimization techniques in Section 4. Section 5 presents a computational complexity analysis of the proposed equalizer and the Viterbi MLSE equalizer, and comparisons are drawn. In Section 6 the uncoded BER performance of the proposed iterative MLSE equalizer is presented for 4QAM and 16-QAM for different channel lengths in frequency selective Rayleigh fading channels. Conclusions are drawn in Section 7.

\footnotetext{
${ }^{1}$ Dept. of Electrical, Electronic and Computer Engineering, University of Pretoria, South Africa. herman.myburgh@up.ac.za

2 Dept. of Electrical, Electronic and Computer Engineering, University of Pretoria, South Africa, and Defense Research, CSIR, Pretoria, South Africa. corne.olivier@eng.up.ac.za
} 


\section{THE ITERATIVE MLSE EQUALIZER}

For systems employing single-carrier modulation in a multipath fading environment, the received symbols are described by [2], [8]

$$
r_{k}=\sum_{j=0}^{L-1} h_{j} s_{k-j}+n_{k},
$$

where $s_{k}$ denotes the $k$ th complex symbol in the transmitted sequence of $N$ symbols chosen from an alphabet $D$ containing $M$ complex symbols. $r_{k}$ is the $k$ th received symbol, $n_{k}$ is the $k$ th Gaussian noise sample, and $h_{j}$ is the $j$ th coefficient of the estimated CIR [5].

To find the most likely transmitted sequence $\mathbf{s}=\left\{s_{1}, s_{2}, \cdots, s_{N}\right\}^{T}$, the cost function [8]

$$
E=\sum_{k=1}^{N}\left|r_{k}-\sum_{j=0}^{L-1} h_{j} s_{k-j}\right|^{2}
$$

needs to be minimized. The Viterbi MLSE equalizer is able to solve this problem exactly, with computational complexity linear in $N$ and exponential in $L$ [8]. The proposed iterative equalizer also minimizes the cost function in (2), with computational complexity linear in $N$ but almost independent of $L$, thus enabling it to perform near-optimal sequence estimation in systems with extremely long CIR lengths with very low computational cost.

\section{A. System Model}

In [9] the authors stated that (2) can be written as

$$
E=-\frac{1}{2} \mathbf{s}^{H} \mathbf{T s}-\mathbf{I}^{H} \mathbf{s}
$$

where $\mathbf{I}$ is a column vector with $N$ elements, $\mathbf{T}$ is a square matrix with $N$ rows and $N$ columns, and $H$ implies the Hermitian transpose. The derivation of the iterative BPSK equalizer proposed in [9] was trivial and (3) could be used as is. When a system employing a complex modulation alphabet is considered, however, (3) needs some treatment to account for the complex terms in the received sequence, the estimated CIR and the most likely transmitted sequence. Therefore, for M-QAM, (3) is rewritten as

$$
E=-\frac{1}{2}\left[\mathbf{s}_{i}^{T} \mid \mathbf{s}_{q}^{T}\left[\begin{array}{cc}
\mathbf{X}_{i} & \mathbf{X}_{q}^{T} \\
\mathbf{X}_{q} & \mathbf{X}_{i}
\end{array}\right]\left[\frac{\mathbf{s}_{i}}{\mathbf{s}_{q}}\right]-\left[\mathbf{I}_{i}^{T} \mid \mathbf{I}_{q}^{T}\left[\frac{\mathbf{s}_{i}}{\mathbf{s}_{q}}\right],\right.\right.
$$

where $\mathbf{s}_{i}$ and $\mathbf{s}_{q}$ denote the estimated in-phase and quadrature sequence vectors respectively, $\mathbf{X}_{i}$ and $\mathbf{X}_{q}$ are square matrices of size $N$, symmetric and banded with the width of the band of non-zero elements determined by $L$, and $\mathbf{I}_{i}$ and $\mathbf{I}_{q}$ are vectors with $N$ elements. Here $T$ denotes the transpose operation. It is clear that (4) is in the form of (3). By expanding (2) and mapping the result to (4), a generalized model can for (4) be derived. $\mathbf{X}_{i}$ is a function of $\boldsymbol{\alpha}=\left\{\alpha_{1}, \alpha_{2}\right.$, $\left.\ldots, \alpha_{L-1}\right\}$, determined by

$$
\alpha_{k}=\sum_{j=0}^{L-k-1} h_{i, j} h_{i, j+k}+\sum_{j=0}^{L-k-1} h_{q, j} h_{q, j+k},
$$

with $k=1,2,3, \ldots, L-1$ and $i$ and $j$ denoting the real and complex components of the CIR coefficients respectively. Similarly $\mathbf{X}_{q}$ is a function of $\boldsymbol{\gamma}=\{$ $\left.\gamma_{1}, \gamma_{2}, \ldots, \gamma_{L-1}\right\}$ determined by

$$
\gamma_{k}=\sum_{j=0}^{L-k-1} h_{q, j} h_{i, j+k}+\sum_{j=0}^{L-k-1} h_{i, j} h_{q, j+k},
$$

where again $k=1,2,3, \ldots, L-1$ and $i$ and $j$ denote the real and complex components of the CIR coefficients. $\mathbf{I}_{i}$ and $\mathbf{I}_{q}$ are functions of the observations $\mathbf{r}=\left\{r_{1}, r_{2}\right.$, $\left.\ldots, r_{N+K-1}\right\}^{T}, \boldsymbol{\alpha}$ and $\boldsymbol{\gamma}$.

For a single-carrier system with a data block of length $N$ and CIR of length $L$, with the data block initiated and terminated by $L-1$ known tail symbols, ${ }^{3}$

$$
\mathbf{X}_{i}=-\left[\begin{array}{cccccc}
0 & \alpha_{1} & \cdots & \alpha_{L-1} & \cdots & 0 \\
\alpha_{1} & 0 & \alpha_{1} & \cdots & \ddots & \vdots \\
\vdots & \alpha_{1} & \ddots & \ddots & \vdots & \alpha_{L-1} \\
\alpha_{L-1} & \vdots & \ddots & \ddots & \alpha_{1} & \vdots \\
\vdots & \ddots & \cdots & \alpha_{1} & 0 & \alpha_{1} \\
0 & \cdots & \alpha_{L-1} & \cdots & \alpha_{1} & 0
\end{array}\right]
$$

and

$$
\mathbf{X}_{q}=-\left[\begin{array}{cccccc}
0 & \gamma_{1} & \cdots & \gamma_{L-1} & \cdots & 0 \\
\gamma_{1} & 0 & \gamma_{1} & \cdots & \ddots & \vdots \\
\vdots & \gamma_{1} & \ddots & \ddots & \vdots & \gamma_{L-1} \\
\gamma_{L-1} & \vdots & \ddots & \ddots & \gamma_{1} & \vdots \\
\vdots & \ddots & \cdots & \gamma_{1} & 0 & \gamma_{1} \\
0 & \cdots & \gamma_{L-1} & \cdots & \gamma_{1} & 0
\end{array}\right]
$$

respectively. Also

$$
\mathbf{I}_{i}=\left[\begin{array}{c}
\lambda_{1}-\rho\left(\alpha_{1}+\gamma_{1}+\cdots+\alpha_{L-1}+\gamma_{L-1}\right) \\
\lambda_{2}-\rho\left(\alpha_{2}+\gamma_{2}+\cdots+\alpha_{L-1}+\gamma_{L-1}\right) \\
\lambda_{3}-\rho\left(\alpha_{3}+\gamma_{3}+\cdots+\alpha_{L-1}+\gamma_{L-1}\right) \\
\vdots \\
\lambda_{L-1}-\rho\left(\alpha_{L-1}+\gamma_{L-1}\right) \\
\lambda_{L} \\
\vdots \\
\lambda_{N-L+1} \\
\vdots \\
\lambda_{N-L+2}-\rho\left(\alpha_{L-1}-\gamma_{L-1}\right) \\
\lambda_{N-2}-\rho\left(\alpha_{3}-\gamma_{3}+\cdots+\alpha_{L-1}-\gamma_{L-1}\right) \\
\lambda_{N-1}-\rho\left(\alpha_{2}-\gamma_{2}+\cdots+\alpha_{L-1}-\gamma_{L-1}\right) \\
\lambda_{N}-\rho\left(\alpha_{1}-\gamma_{1}+\cdots+\alpha_{L-1}-\gamma_{L-1}\right)
\end{array}\right]
$$

\footnotetext{
${ }^{3}$ The tail symbols are equal to $0.7071+\mathrm{j} 0.7071$.
} 
and

$$
\mathbf{I}_{q}=\left[\begin{array}{c}
\omega_{1}-\rho\left(\alpha_{1}-\gamma_{1}+\cdots+\alpha_{L-1}-\gamma_{L-1}\right) \\
\omega_{2}-\rho\left(\alpha_{2}-\gamma_{2}+\cdots+\alpha_{L-1}-\gamma_{L-1}\right) \\
\omega_{3}-\rho\left(\alpha_{3}-\gamma_{3}+\cdots+\alpha_{L-1}-\gamma_{L-1}\right) \\
\vdots \\
\omega_{L-1}-\rho\left(\alpha_{L-1}-\gamma_{L-1}\right) \\
\omega_{L} \\
\vdots \\
\omega_{N-L+1} \\
\vdots \\
\omega_{N-L+2}-\rho\left(\alpha_{L-1}+\gamma_{L-1}\right) \\
\omega_{N-2}-\rho\left(\alpha_{3}+\gamma_{3}+\cdots+\alpha_{L-1}+\gamma_{L-1}\right) \\
\omega_{N-1}-\rho\left(\alpha_{2}+\gamma_{2}+\cdots+\alpha_{L-1}+\gamma_{L-1}\right) \\
\omega_{N}-\rho\left(\alpha_{1}+\gamma_{1}+\cdots+\alpha_{L-1}+\gamma_{L-1}\right)
\end{array}\right],
$$

where $\rho=0.7071$ and $\lambda=\left\{\lambda_{1}, \lambda_{2}, \ldots, \lambda_{N}\right\}$ is determined by

$$
\lambda_{k}=\sum_{j=0}^{L-1} r_{i, j+k} h_{i, j}+\sum_{j=0}^{L-1} r_{q, j+k} h_{q, j},
$$

and $\boldsymbol{\omega}=\left\{\omega_{1}, \omega_{2}, \ldots, \omega_{N}\right\}$ is determined by

$$
\omega_{k}=\sum_{j=0}^{L-1} r_{q, j+k} h_{i, j}+\sum_{j=0}^{L-1} r_{i, j+k} h_{q, j},
$$

where $k=1,2,3, \ldots, N$ with $i$ and $q$ again denoting the real and complex components of the respective vectors.

\section{B. Iterative MLSE Equalization}

It was shown in [10] that (3) is a Lyapunov function in the high gain limit, where the neuron threshold function approaches a sgn-function, for the dynamic system given by

$$
\frac{d \mathbf{u}}{d t}=-\frac{\mathbf{u}}{\tau}+\mathbf{T s}+\mathbf{I}
$$

where $\tau$ is an arbitrary constant and $\mathbf{u}=\left\{u_{1}, u_{2}, \ldots\right.$, $\left.u_{N}\right\}$. Starting from a zero initial state, the system will converge to a steady state denoted $\mathbf{u}^{*}$ so that $\mathbf{s}^{*}$, a function of $\mathbf{u}^{*}$, will minimize the cost function $E$ in (3). $\mathbf{s}^{*}$ is therefore the MLSE sequence estimate.

An iterative solution for (13) is given by

$$
\begin{aligned}
& \mathbf{u}^{n+1}=\mathbf{T} \mathbf{s}^{n}+\mathbf{I} \\
& \mathbf{s}^{n+1}=g\left(\beta \mathbf{u}^{n+1}\right)
\end{aligned},
$$

where $n$ indicates the iteration number and $g\left(\beta u_{k}\right)$, where the gain $\beta=1$, is the decision function. After a few iterations the source symbol estimates are read from s. For the proposed two dimensional M-QAM equalizer, $\mathbf{u}=\left[\mathbf{u}_{i}^{T} \mid \mathbf{u}_{q}^{T}\right], \mathbf{s}=\left[\mathbf{s}_{i}^{T} \mid \mathbf{s}_{q}^{T}\right], \quad \mathbf{I}=\left[\mathbf{I}_{i}^{T} \mid \mathbf{I}_{q}^{T}\right]$, and $\mathbf{T}=\left[\begin{array}{ll}\mathbf{X}_{i} & \mathbf{X}_{q}^{T} \\ \mathbf{X}_{q} & \mathbf{X}_{i}\end{array}\right]$.

\section{MULTILEVEL NEURON}

The BPSK equalizer proposed in [9] uses a bipolar neuron to estimate the transmitted symbols. For M-QAM modulation, however, there are multiple signal levels per dimension for $M>2$. Therefore a neuron with multiple decision levels has to be used. A multilevel neuron is realized by adding multiple bipolar sigmoid functions, each shifted by a certain amount. The multilevel neuron used for the iterative 16-QAM equalizer is given by

$$
g(u)=0.667\left(\sum_{k=-1}^{1} \frac{1}{1+e^{-\beta(u+\theta k)}}\right)-1,
$$

where $\beta$ is a scaling factor used for optimization, and $\theta$ is the value by which the neurons are shifted, determined by

$$
\theta=\sqrt{\left(\sum_{k=0}^{L-1} h_{i, k}^{2}\right)^{2}+\left(\sum_{k=0}^{L-1} h_{q, k}^{2}\right)^{2}} .
$$

Fig. 1 shows the multilevel neuron described by (15) for $\beta=1$ and $\theta=15$.

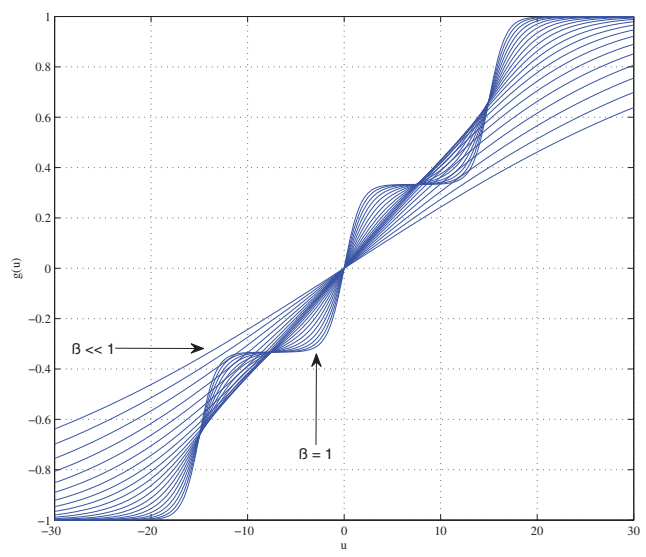

Fig. 1 - The multilevel neuron for $Z=1$ to $Z=20$.

\section{IV.OPTIMIZATION}

As the system iterates, $\beta$ is updated systematically according to the exponential function

$$
\beta=5^{\frac{2(n-Z+1)}{Z}}
$$

where $Z$ denotes the number of iterations and $n$ denotes the iteration number, to ensure that the system converges to a near-optimal local minimum in 
the solution space. This causes $\beta$ to start at a near-zero value and to exponentially converge to 1 with each iteration. This process is called simulated annealing [11]. The effect of annealing on the neurons during the iteration cycle is shown in Fig. 1, with the slope of the multilevel neuron increasing as $\beta$ is updated with each iteration. Simulated annealing, together with asynchronous neuron updates, ensure near-optimal sequence estimation. These techniques allow the system to escape less optimal local minima in the solution space, leading to increased system performance.

\section{COMPUTATIONAL COMPLEXITY}

It is assumed that a block of $N$ transmitted symbols $\mathbf{s}=$ $\left\{s_{1}, s_{2}, \ldots, s_{N}\right\}^{T}$ is subjected to ISI in a multipath fading channel with a CIR length of $L$ and is equalized by the proposed equalizer. The equalizer requires $Z$ iterations. ${ }^{4}$ For a M-QAM modulation alphabet, ${ }^{5}$ the computational complexity of the proposed MLSE equalizer is $4 Z N(N+3)+8 L(N+1)+2 L^{2}{ }^{6}$ The Viterbi MLSE equalizer has computational complexity proportional to $N M^{(L-1)}$. Fig. 2 shows the computational complexity of the proposed equalizer and a 16-QAM Viterbi MLSE equalizer for $L=2$ to $L=6$ for a data block length of $N=200$. Also, Fig. 3 shows the computational complexity of the proposed equalizer for $L=2$ to $L=1000$ for a data block length of $N=1000$.

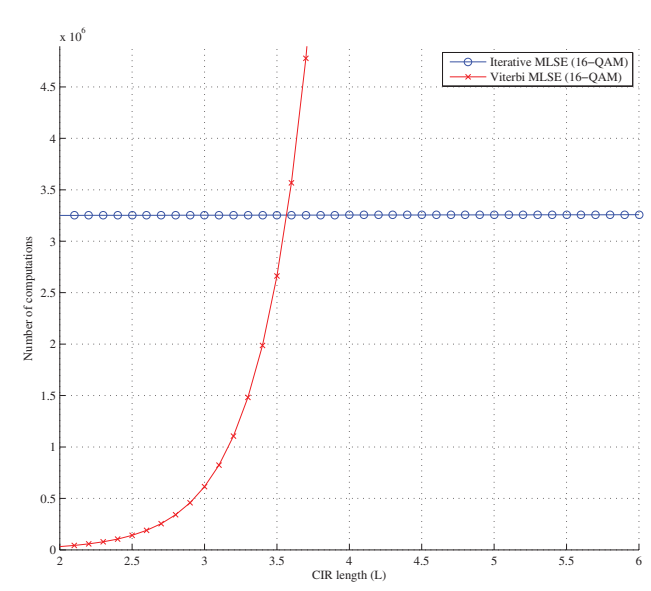

Fig. 2 - Complexity comparison for $L=2$ to $L=6$.

For communication systems with large delay spreads, and therefore with large $L$, it is clear that the computational complexity of the proposed equalizer is

\footnotetext{
${ }^{4} \mathrm{Z}=20$ iterations are used.

5 The complexity is independent of the modulation alphabet size for any M-QAM equalizer realized with this model, for $\mathrm{M}>2$. For $\mathrm{M}=2$ (BPSK) the complexity is halved.

${ }^{6}$ For $N \gg>$ the contribution of $L$ becomes negligible.
}

almost independent of the CIR length $L$, therefore enabling it to equalize signals in systems with extremely long memory seamlessly, while offering near-optimal performance. The computational significance of the proposed equalizer is due to the high parallelism of its underlying neural network structure [10].

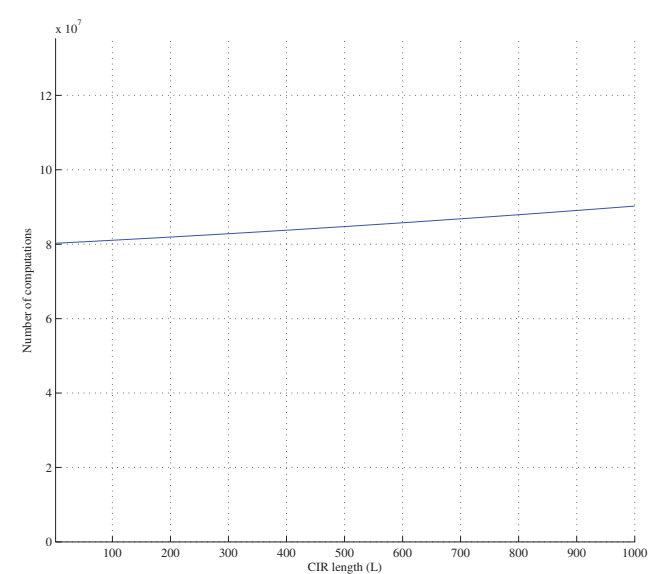

Fig. 3 - Complexity of the proposed equalizer for $L=2$ to $L=1000$.

\section{NUMRERICAL RESULTS}

The proposed equalizer was evaluated for uncoded 4QAM and 16-QAM modulated systems with extremely long memory. ${ }^{7}$ To simulate the fading effect on each tap, the Rayleigh fading simulator proposed in [12] was used to generate uncorrelated fading vectors. In all simulations the nominal CIR weights were chosen as such that $\mathbf{h}^{T} \mathbf{h}=1$, where $\mathbf{h}$ is a column vector of length $L$. Least Squares (LS) channel estimation was used to determine the CIR for the 4-QAM system, in order to include the effect of imperfect CSI in the simulation results. Perfect CSI was assumed for the 16-QAM system, by taking the average of each fading vector to construct the CIR vector for each data block, to demonstrate optimal reconstruction of transmitted symbols from the ISIcorrupted received symbols. The equalizers were simulated at a carrier frequency $f_{c}=900 \mathrm{MHz}$ with a symbol period of $T_{s}=3.7 \mu \mathrm{s}$, a mobile speed of $v=3$ $\mathrm{km} / \mathrm{h}$ and $Z=20$ iterations, for various CIR lengths, for all scenarios. ${ }^{8}$

The 4-QAM equalizer was simulated firstly for short CIR lengths and compared to the Viterbi MLSE equalizer's performance. A data block length of $N=$

\footnotetext{
7 The only difference between the 4-QAM and 16-QAM equalizers is the decision function. 4-QAM uses a bipolar neuron; 16-QAM uses the multilevel neuron in (15).

${ }^{8}$ Although the simulations were performed in baseband, the settings of $f_{c}, T_{s}$, and $v$ were used to generate realistic Rayleigh fading vectors.
} 
200 was used and the CIR lengths were chosen as $L=$ 2, $L=6$, and $L=10$, and $0.3 N$ pilot symbols were used for channel estimation. Fig. 4 shows the BER graphs for these simulations.

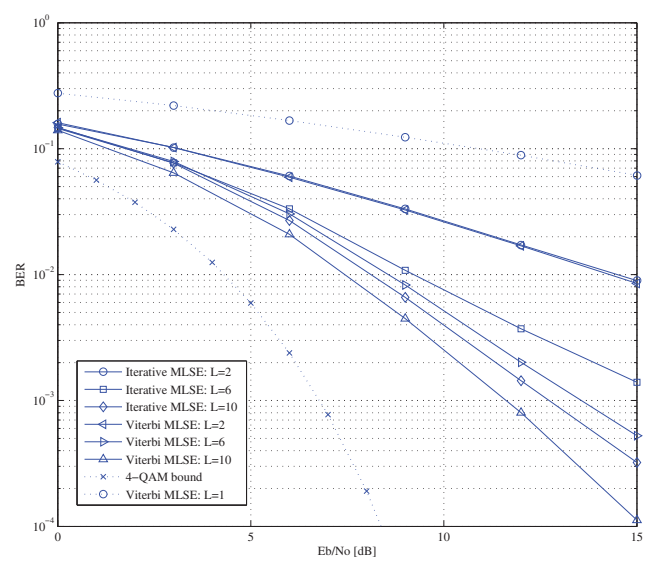

Fig. 4 - 4-QAM performance for CIR lengths 2, 6 and 10 for Rayleigh fading channels.

It is clear that the Viterbi MLSE equalizer outperforms the 4-QAM equalizer for $L=6$ and $L=$ 10. It was concluded in [9] that the Viterbi MLSE equalizer performs better in short channels than the iterative BPSK equalizer propose therein, of which the 4-QAM equalizer is an extension.

The 4-QAM equalizer was also evaluated for long CIR lengths $L=20, L=50, L=100$, and $L=200$, for a data block of length $N=1000$ and $3 L$ training symbols for channel estimation. Fig. 5 shows the performance of the 4-QAM equalizer. From Fig. 5 it is clear that the equalizer effectively equalizes the signals corrupted by hundreds of interfering symbols. Note that this would have required $4^{199}$ states per transmitted symbol for $L=200$ had the Viterbi MLSE equalizer been used.

The iterative 16-QAM MLSE equalizer was simulated for long channel lengths only, since its performance in short channels is not comparable to that of the Viterbi MLSE equalizer. The equalizer was evaluated for various CIR lengths from $L=50$ to $L=$ 400 , corresponding to delay spreads of $185 \mu$ s to 1.48 $\mathrm{ms}$, for a data block length of $N=500$. The simulation results are shown in Fig. 6 and it is clear that proposed equalizer effectively equalizes the corrupted symbols.

Fig. 7 shows the convergence of the estimated symbols in the 16-QAM equalizer for each iteration during equalization for $L=50$ at $\mathrm{E}_{\mathrm{b}} / \mathrm{N}_{0}=14 \mathrm{~dB}$. For extremely long channels, the iterative MLSE equalizer optimally recombines the energy of ISI-corrupted received signal, producing optimal 16-QAM performance without the use of error-correction coding. This is a significant result. Here the Viterbi MLSE equalizer would have required $16^{399}$ states per transmitted symbol for $L=400$; clearly an impossible task.

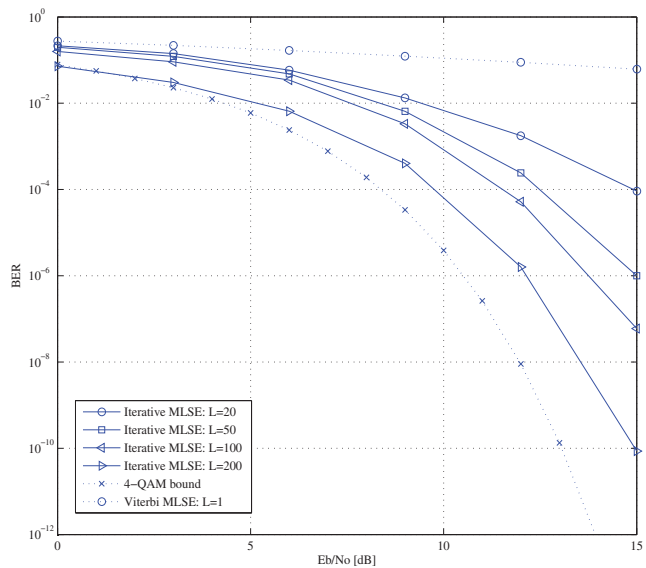

Fig. 5 - 4-QAM performance for CIR lengths 20, 50, 100, and 200 for Rayleigh fading channels.

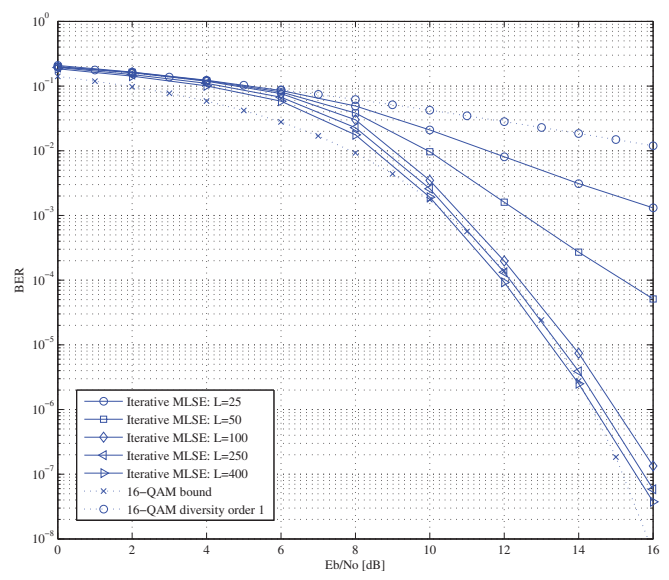

Fig. 6 - 16-QAM performance for CIR lengths 50, 100, 250 and 400 for Rayleigh fading channels.

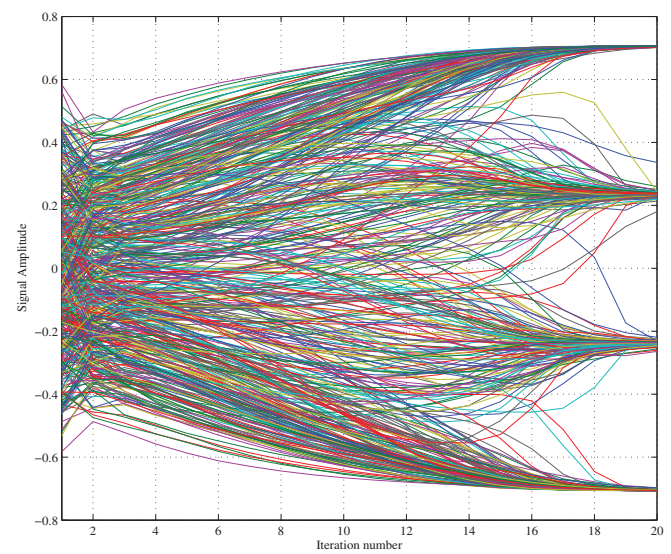

Fig. 7 - Convergence of the estimated symbols in the 16QAM equalizer for $N=500$ and $L=50$. 


\section{CONCLUSION}

In this paper a neural network based low complexity iterative M-QAM MLSE equalizer was presented and evaluated by computer simulation in a frequencyselective Rayleigh fading environment. Results showed that the performance of the proposed equalizer does not match that of the Viterbi MLSE in short channels, but it was clear that for both the 4-QAM and 16-QAM versions, the equalizer was able to effectively equalize signals corrupted by hundreds of interfering symbols. The computational complexity analysis showed that the proposed M-QAM equalizer has superior complexity over conventional optimal equalizers, for single-carrier systems with moderate to high bandwidth, in that it has computational complexity linear in the data block length and almost independent of the channel memory length, due to the high parallelism of its underlying neural network structure. The approximate independence of the complexity from the channel memory is significant, as the CIR length is the dominant term in the complexity of all optimal equalizers. Also, the complexity of any M-QAM equalizer realized with the proposed model will be identical for $\mathrm{M}>2$, as the complexity is dependent only on the data block length and the number of dimensions, and only weakly dependent on the channel memory length. The proposed iterative MQAM MLSE equalizer can be applied to a number of wireless communication systems, among which underwater-, CDMA- , and multiple transmit antenna communication systems are being investigated.

\section{REFERENCES}

[1]. Viterbi A.D. Error bounds for convolutional codes and an asymptotically optimum decoding algorithm, IEEE Transactions on Information Theory 13 (1) (1967). pp. 260-269.

[2]. J.G. Proakis. Digital Communications, 4th edition. McGraw-Hill. New York, 2001.

[3]. Bahl L. Cocke J. Jelinek F. Raviv J. Optimal decoding of linear codes for minimizing symbol error rate, IEEE Transactions on Information Theory 20 (3) (1974). pp. 284-287.

[4]. Dual-Hallen A. Hegaard C. Delayed decision feedback sequence estimation, IEEE Transactions on Communications 37 (5) (1989). pp. 428-436.

[5]. Gerstacker W.H. and Schober R. Equalization concepts for EDGE, IEEE Transactions on Wireless Communications 1 (1) (2002). pp. 190 - 199.

[6]. Terry J. Heiskala J. OFDM Wireless LANs: A Theoretical and Practical Guide. Sams Publishing. Indianapolis, IN, 2001.

[7]. Koetter R. Singer A.C. Tüchler M. Turbo Equalization, IEEE Signal Processing Magazine 21 (1) (2004). pp. 67-80.

[8]. Forney G.D. The Viterbi Algorithm, Proceedings of the IEEE, 61 (3) (1973). p. 268-278.

[9]. Myburgh H.C. Olivier J.C. Near-Optimal Low Complexity MLSE Equalization. Proceedings of the Conference "Wireless Communications and Networking Conference (WCNC 2008)", Las Vegas,
NV, USA, 31 March - 3 April 2008, pp. 226-230.

[10].Hopfield J.J. Tank D.W. Neural computations of decisions in optimization problems, Biology and Cybernetics 52 (1985). pp. 1-25.

[11].Russell S. Norvig P. Artificial Intelligence: A Modern Approach, 2nd edition. Prentice Hall, New Jersey, 2003.

[12].Y.R. Zheng and C. Xiao. Improved models for the generation of multiple uncorrelated Rayleigh fading waveforms, IEEE Communications Letters 6 (2002). pp. 256-258.

J.C. Olivier is an Exceptional Professor at the University of Pretoria and a Principle Scientist at the CSIR in Pretoria, South Africa. He was with Nokia Research in the USA and with Bell Northern Research in Canada until 2003. His research interests include estimation and detection theory as well as machine learning. He is an Associate Editor for the IEEE Transactions on Wireless Communications and TPC co-chairman for ICC 2010.

H.C. Myburgh received his B.Eng. (Computer Engineering) degree (cum laude) from the University of Pretoria, South Africa, in 2007. He is currently studying towards his M.Eng degree in Electronic Engineering, majoring in Digital Communication Systems, with a special interest in equalization and error control coding. He is currently holding a position as a junior lecturer at the University of Pretoria, South Africa, and he is also the chairman of the University of Pretoria IEEE Student Branch for the year of 2009. 